\title{
The role of microendodontics in the treatment of difficult cases such as C-shaped orifice of mandibular second molar with 5 canals
}

\author{
Harry Huiz Peeters
}

Private Practicing

\begin{abstract}
Finding the incidence of anatomical variation in root canals is commonly found as a result of more sophisticated diagnostic tools. Therefore, a thorough understanding of the normal anatomy of canals and its variations in root canal treatments would be helpful in achieving the desired result. Usual root configuration of a mandibular second molar is two separate roots, distal and mesial, with one canal each. The incidence of a distinct 4 root canal in the distal root in $\mathrm{C}$ - shaped orifices is a rare phenomenon that is reported in literature. This case study shows how to locate and manage a rare anatomical variation of mandibular second molar with a distinct 4 canal in distal aspect in a C-shaped orifice successfully, the successful treatment of this case explains that we should have an awareness and understanding of the presence of additional canals and abnormal morphology of the canals. Beside that the operator's perseverance and patience as well as sophisticated diagnostic tools such as the dental operating microscope, ultrasonic tips have an important role in treating unusual configurations of canals.
\end{abstract}

Key words: Dental operating microscope, C-shaped orifices, ultrasonic tips

\section{INTRODUCTION}

The basic precondition for successful root canal treatment is to seal the root canal system completely through a thorough mechanical and chemical debridement of the entire root canal followed by 3 dimensional obturation with an inert filling material and a final coronal restoration, thereby preventing from reinfection of microorganism. ${ }^{1}$ One of the root canal treatment failures is the presence of microorganism in the root canal system, because of insufficient debridement of the entire root canal system especially in the complex root canal system such as C- shaped canal. ${ }^{2}$
The complexity of the root canal system has both technical and microbiological objectives that should be overcome. The most frequent reason for failure is oversight of their existence. Therefore, a thorough understanding of canal anatomy in treating root canals which have anatomical variations and racial characteristic groups is an important prerequisite in negotiating and determinating the location of a canal as well as its subsequent management. ${ }^{3}$

Finding the incidence of anatomical variations in daily practice becomes more frequent due to the development of diagnostic tools. This case study shows how to locate and manage a rare anatomical variation successfully, especially in 
treating mandibular second molar with a distinct 4 canal in distal aspect in a C-shaped orifice.

\section{CASE REPORT}

A 65-year-old female patient came to our clinic with a chief complaint of intermittent pain in relation to tooth $\# 47$ (mandibular right second molar), especially when she closed her mouth. That particular pain had lasted over a 6 months period, and had increased in intensity over the previous 2 days. Medical history was noncontributory. Intraorally, the patient had moderate oral hygiene. Tooth \#47 was non-responsive to cold and electric pulp test. Further examination showed that tooth \#47 was tender to percussion and non-responsive to palpation, and there was an intact big composite restoration.

There was no evidence of either swelling or sinus tract. The periodontal condition was excellent, with no gingivitis and absence of pocket. Radiographic evaluation of the involved tooth showed no evidence of radiolucency and has already been treated endodontically. The tooth was diagnosed as a pulpless tooth with an infected root canal system. Root canal treatment needed be done as a treatment of choice, followed by restoration and crowning. The patient signed the informed consent as a medico-legal procedure. The patient would be reviewed after 6 months and then annually. At the first visit, the tooth was isolated under rubber dam, accessed with anesthesia and sterilized by iodine solution. Under microscope inspection, composite restoration and caries dentine were removed prior to accessing the pulp chamber by fissure round bur and straight line access was achieved. It was discovered that tooth \#47 had a C-shaped orifice. Using a size 0.8 $\mathrm{C}+$ file a canal was identified in each of the mesial and distal roots. Further microscopic investigation (under magnification 1x) of the distal root orifice revealed possible others canals in the distal aspect. When explored with size $0,8 \mathrm{C}+$ file, there were 4 canals in distal aspect. All were confirmed radiographically. It was concluded that tooth \#47 had 5 canals. An apex locator was used to verify the working length in all five root canals. An additional operative radiograph was taken to confirm the independent presence of the four canals in the distal root. Individual canal instrumentation was performed sequentialy with rotary Ni-Ti Protaper Universal files nos: 15, 20, 25, $30(6 \%)$ using a crown-down pressureless technique to a master apical size 30. Copious chemical irrigation was performed with $2,5 \%$ sodium hypochlorite. After completion of chemo-mechanical preparation, ultrasound was used to activate the irrigants in the entire canals, this has been proved as an valuable action in penetrating small spaces. The root canals were dried with sterile paper point, the pulp chamber was examined again under the microscope for any additional canals and photographs were taken under magnification $1.6 \mathrm{x}$ (Fig. 1) and an intracanal dressing of Ultracal paste was used before placing temporary filling.

During the second visit the patient had no symptoms. Obturation was performed at this second appointment using warm vertical compaction of gutta-percha and AH-26 as a root canal sealer. Prior to obturation, EDTA (17\%) was used to removed the smear layer and a final irrigation with 2,5\% sodium hypochlorite followed. Excess gutta-percha cones were cut at the level of the root canal orifices and vertical compaction was applied immediately with ISO size 40 finger plugger after heating by System-B, this action will ensure better homogeneity of gutta-percha and the gutta-percha will fill of canal irregularties, accessory canal. A coronal permanent restoration of composite was placed and a postoperative radiograph was taken in order to assess the quality of obturation in all canals. The patient was requested to return postoperatively after 6 months and again after one year. At the recall appointment, the patient was symptom free.

\section{DISCUSSION}

Success in root canal therapy was originally base on cleaning, shaping, and filling the entire root canal system effectively. Achieving a successful result, a thorough knowledge of the morphology of the root canal system is an essential prerequisite. Many methods are used to investigate the root canal anatomy in vitro and in vivo, however, the specimens are destroyed by grinding or splitting. Based on these concerns, radiography becomes the most practical and often used method to predict the root canal anatomy in both laboratory and clinical studies. Careful 


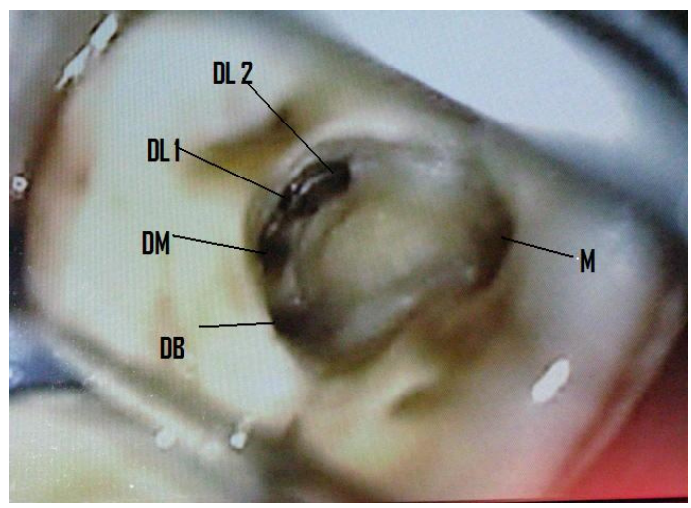

Figure 1. Intraoperative photograph of the pulp chamber floor revealing 4 separate orifices in distal aspect. (DB, DM, DL1, DL2).

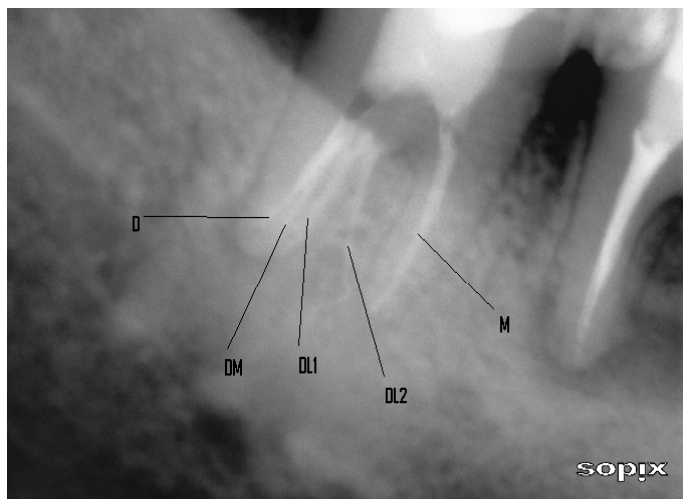

Figure 2. Postoperative radiograph.

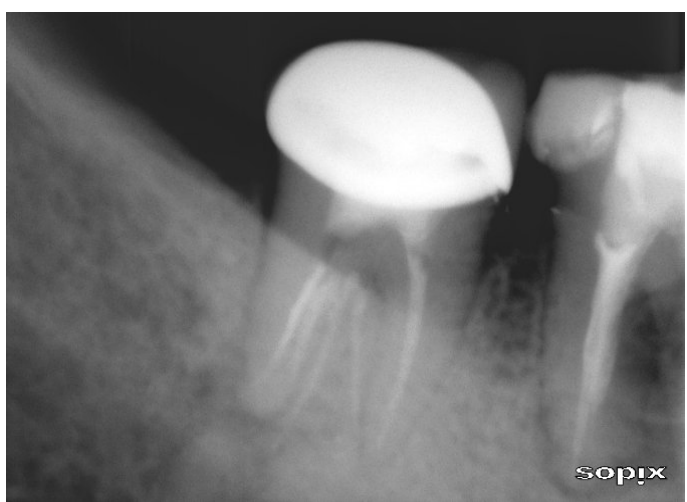

Figure 3. After 6 months recall, canals were filled with gutta-percha.

assessment of the preoperative radiograph is a key step for subsequent root canal treatment, such as root canal preparation and obturation. Preoperative radiographs may aid in visualizing and observing the anatomy of various root canal systems, especially the complicated ones, such as a C-shaped canal configuration. A C- canal system is a canal variant mostly seen in mandibular second molars. ${ }^{4}$ The incidence of $\mathrm{C}$-shaped molars in the general population is approximately 8 percent, but can be as high as 31 percent in Asian ethnic groups. ${ }^{5}$ The anatomy of teeth is not always shaped normal. A great number of variations can occur in formation, number of roots, and their shape. However, most dentists must have an awareness of the presence of rare variation anatomy; even thought abnormalities are rare. ${ }^{6}$

Many studies have been performed over the years to investigate tooth morphology, including mandibular second molars. ${ }^{1}$ The major variant in this group is the madibular first molar with 3 roots. The additional root is usually located on the lingual aspect ${ }^{7}$, whereas the most prominent prevalence of $\mathrm{C}$-shaped root canals is reported in the mandibular second molar. ${ }^{8}$

Yang' claimed that there was a high prevalence of $\mathrm{C}$-shaped roots in mandibular second molars $(31.5 \%)$, in which $68.3 \%$ of the teeth had C-shaped canal orifices. ${ }^{9}$ The usual root configuration of a mandibular second molar is two separated roots, mesial and distal. The distal root usually has one canal and the mesial root has two canals that often converge in the apical area. ${ }^{10}$ In general, two-rooted mandibular second molar have a single distal canal (about $90 \%$ ) and two mesial canals (more than $70 \%$ ). ${ }^{11}$

C-shaped molar configuration develops when Hertwig's epithelial root sheath fails to fuse either on the buccal or lingual surface. That fusion failure on both the buccal and lingual aspect would results in a groove on the opposite side of the root that is present coronoapically. ${ }^{11}$ The canal configuration was significantly related to race, with more Asian having $\mathrm{C}$ - shaped canals. ${ }^{11}$

Therefore, recognizing anatomical variation of C-shaped canal configuration would be helpful in determinating and locating canal orifices preventing catastrophic perforations when instrumenting the thin isthmus. Because of this complex morphology of second mandibular molar more over the presence of a high incidence of transverse anatomoses, lateral canal and apical deltas make it difficult to clean and seal the canal system adequately. In this case report to minimize the failure of sealing the canal, the treatment was done with warm vertical compaction that allows the gutta-percha flows into small spaces, deltas, anastomoses and lateral canals effectively and will ensure better homogeneity of gutta-percha. 
The ultrasonic device was used that is believed to be very effective to provide optimal cleaning conditions.

\section{CONCLUSION}

The main goal of this case study is to report a successful treatment of 4 distinct root canals in distal in the $\mathrm{C}$-shaped orifices. The success of nonsurgical root treatment of mandibular second molar with 5 root canals is a result of the combination of knowledge of anatomical variations and experience as well as the support of sophisticated diagnostic tools such as dental operation microscope and ultrasonic device.

\section{REFERENCES}

1. Vertucci FJ. Root canal morphology and its relationship to endodontic procedures. Endodontic topics 2005;10:3-29.

2. Nair PN. On the causes of persistent apical periodontitis: A review. Int Endod J 2006; 39:249-81.

3. Plotino G. A Mandibular third molar with three mesial roots: A case report. J Endod 2008;34:224-6.

4. Fan B, Gao Y, Fan W, Gutmann J. Identification of a $\mathrm{C}$ - shaped canal system in mandibular second molars-Part II: The effect of bone image superimposition and intraradicular contrast medium on radiograph interpretation. J Endod 2008;34:160-5.

5. Cooke HG, Cox FL. C-shaped canal configurations in mandibular molar. J Am Dent Assoc 1979;99:836-9.

6. Jamileh G, Neda N, Mina Z, Ehsan R. Mandibular first molar with four distal canals. J Endod 2007;33:1481-3.

7. Speber GH, Moreau JL. Study of the number of roots and canals in Senegalese first permanent mandibular molars. Int Endod J 1998;31:11722.

8. Weine FS. The C- shaped mandibular second molar: incidence and other considerations. J Endod 1998;24:372-5.

9. Yang ZP, Yang SF, Lin YC, Shay C, Chi CY. Cshaped root canals in mandibular second molar in a Chinese population. Endod Dent Traumatol 1988;4:160-3.

10. Yi Min, Bing Fan, Gary SP, Cheung, James L, Gutmann, Mingwen Fan. C-shaped canal system in mandibular second molars. Part III: The morphology of the pulp chamber floor 2006;32:1155-9.

11. Manning SA. Root canal anatomy of mandibular second molars. Part II: C-shaped canals. Int Endod J 1990;23:40-5. 